\title{
The Chandra X-ray Spectrum of the 10.6 s Pulsar in Westerlund 1: Testing the Magnetar Hypothesis
}

\author{
S.L. Skinner \\ CASA, Univ. of Colorado, Boulder, CO 80309-0389 \\ R. Perna \\ JILA, Univ. of Colorado, Boulder, CO 80309-0440 \\ S.A. Zhekov \\ Space Research Institute, Moskovska str. 6, Sofia-1000, Bulgaria
}

\begin{abstract}
Two sensitive Chandra X-ray observations of the heavily-reddened galactic starburst cluster Westerlund 1 in May and June 2005 detected a previously unknown X-ray pulsar (CXO J164710.20-455217). Its slow 10.6 s pulsations, moderate $\mathrm{X}$-ray temperature $\mathrm{kT} \approx 0.5 \mathrm{keV}$, and apparent lack of a massive companion tentatively suggest that it is an Anomalous X-ray Pulsar (AXP). An isothermal blackbody model yields an acceptable spectral fit but the inferred source radius is much less than that of a neutron star, a result that has also been found for other AXPs. We analyze the X-ray spectra with more complex models including a model that assumes the pulsar is a strongly magnetized neutron star ("magnetar") with a light element atmosphere. We conclude that the observed X-ray emission cannot be explained as global surface emission arising from the surface of a cooling neutron star or magnetar. The emission likely arises in one or more localized regions ("hot spots") covering a small fraction of the surface. We discuss these new results in the context of both accretion and magnetar interpretations for the X-ray emission.
\end{abstract}

Subject headings: open clusters and associations: individual (Westerlund 1) — stars: formation — stars: neutron — X-rays: stars

\section{Introduction}

X-ray pulsars most commonly occur as members of binary systems and their primary energy source is believed to be accretion from a donor star onto the neutron star. However, 
about a half dozen X-ray pulsars have been identified over the past decade that do not show the telltale Doppler shifts associated with orbital motion and have slow pulsation periods of $\approx 6-12 \mathrm{~s}$ and similar X-ray properties. They are now known collectively as Anomalous X-ray Pulsars (AXPs). The apparent absence of a donor star, slow pulsation periods, and X-ray luminosity levels of AXPs suggest that their X-ray emission is not powered by binary accretion or rotational energy loss. Two somewhat different pictures have emerged to explain the X-ray emission. It has been proposed that AXPs are single neutron stars with intense magnetic fields $\mathrm{B} \gtrsim 10^{14} \mathrm{G}$ known as "magnetars" (Duncan \& Thompson 1992) whose energy release via magnetic field decay heats the neutron star surface to X-ray emitting temperatures (Thompson \& Duncan $1996=$ TD96). This idea is attractive but a direct measurement to confirm the presence of the very high magnetic field strengths postulated for magnetars is still needed. A second possibility is that the X-ray emission may be due to accretion from a residual disk (van Paradijs et al. 1995; Chatterjee, Hernquist, \& Narayan 2000; Alpar 2001).

The X-ray properties of AXPs have been summarized by Mereghetti et al. (2002 = M02) and Kaspi \& Gavriil $(2004=$ KG04). AXPs have moderately soft X-ray spectra and X-ray luminosities in the range $\mathrm{L}_{\mathrm{X}} \sim 10^{34}-10^{35} \mathrm{ergs} \mathrm{s}^{-1}$. Acceptable spectral fits generally require two components. In most cases the emission is modeled with a thermal (e.g. blackbody) component at $\mathrm{kT}_{b b} \approx 0.4-0.6 \mathrm{keV}$ plus a harder power-law component. A wide range of photon power-law indices $\alpha_{p h} \approx 2$ - 5 has been reported in the literature (M02, Perna et al. $2001=\mathrm{P} 01)$. The origin of the non-thermal power-law emission is uncertain but it is thought to arise in the magnetosphere (M02).

The interpretation of the cool thermal X-ray emission as integrated blackbody emission from the surface of the neutron star is problematic for AXPs because the source radius inferred from isothermal blackbody fits is usually only a small fraction of the radius of a neutron star $\left(\mathrm{R}_{n s} \approx 10 \mathrm{~km}\right)$. This result could be explained if the intrinsic surface emission is masked by more luminous X-ray emission originating in hot spots that cover only a small fraction of the stellar surface. Gotthelf \& Halpern $(2005,=$ GH05) have suggested that this is the case for the emission of the transient AXP XTE J1810-197 monitored during the decay phase of a recent outburst. They model its emission as a concentric spot arrangement using a two-temperature blackbody model and argue that this model is more physically meaningful for XTE J1810-197 than the usual blackbody + power-law interpretation.

The spot interpretation offers a promising means of explaining the variable X-ray emission of transient AXPs such as XTE J1810-197 but the origin of quiescent emission from AXPs is another question. The issue of small source radii inferred from blackbody models of quiescent AXPs was explored by P01. They noted that the intrinsic X-ray spectrum 
of a magnetar will differ from that of a simple blackbody due to the effects of anisotropic heat flow through the envelope, reprocessing of photons by a light-element atmosphere, and general relativistic corrections. Thus, source radii inferred from blackbody fits of magnetars may be incorrect. They developed a sophisticated magnetar model incorporating the above effects and used it to fit the $A S C A$ X-ray spectra of five AXPs. The source radii inferred from their magnetar models were generally consistent with the radius of a neutron star, but interestingly their spectral fits still required a power-law component. The X-ray temperatures determined from their magnetar models were $\mathrm{kT}_{\text {magnetar }} \approx 0.3-0.4 \mathrm{keV}$, which are slightly lower than the blackbody temperatures but still higher than expected for thermal cooling alone.

Since only a handful of AXPs are presently known, additional objects are needed to fully characterize their X-ray properties and guide the development of realistic X-ray emission models. We analyze here the X-ray spectrum of a new $10.6 \mathrm{~s}$ pulsar that was serendipitously detected in two sensitive Chandra observations of the galactic starburst cluster Westerlund 1 (Wd1) in May - June 2005 (Skinner et al. $2005=$ S05; Muno et al. $2006=$ M06; Skinner et al. $2006=\mathrm{S} 06$ ). This object shows several of the characteristic X-ray properties of AXPs including slow pulsations, a thermal component temperature $\mathrm{kT} \approx 0.5-0.6 \mathrm{keV}$, a possible power-law contribution, and an inferred emitting area from isothermal blackbody fits that is much less than the surface area of a neutron star. NTT images show no evidence for an infrared counterpart down to a limiting magnitude $\mathrm{K}_{s}=18.5$ (S05, M06) which initially seems to rule out a massive $\left(>1 \mathrm{M}_{\odot}\right)$ companion and thus strengthens the AXP classification. Even so, thermal emission models indicate that the Wd 1 pulsar is less luminous in X-rays than other AXPs based on current distance estimates.

It has recently been argued that the $10.6 \mathrm{~s}$ pulsar in $\mathrm{Wd} 1$ is a magnetar (M06). However, further observational work will be needed to confirm this hypothesis. The existing constraint on the spin-down rate from two Chandra observations spaced $\approx 1$ month apart is not yet sufficient to rule out spin-down as the X-ray energy source. In addition, more sensitive optical/IR/millimeter searches are necessary to determine if a low-mass companion or residual disk are present. It is our objective here to examine more sophisticated X-ray spectral models for the Wd 1 pulsar that go beyond the isothermal blackbody model considered in earlier work. Most importantly, we consider a model that assumes the neutron star is a magnetar and show that the source radius inferred from the magnetar model is still much less than that of a neutron star. This result gives support to the idea that the detected X-ray emission originates mainly in one or more localized regions (hot spots) covering only a small fraction of the neutron star surface. Such spots could form on the surface of a magnetar but might also occur as heated polar caps on a conventional X-ray pulsar. 


\section{Chandra Observations and Data Reduction}

Chandra observed Westerlund 1 with the ACIS-S imaging array on 22-23 May 2005 and 18-19 June 2005 with exposure live times of $18,808 \mathrm{~s}$ and $38,473 \mathrm{~s}$, respectively. Additional observational details are given in S06. The observation was obtained in timed faint-event mode using a $3.2 \mathrm{~s}$ frame time. For the spectral analysis discussed below, Level 1 event files provided by the Chandra X-ray Center were processed with CIAO ${ }^{1}$ (vers. 3.3) to generate an updated Level 2 event file that takes into account the latest gain and effective area calibrations (CALDB vers. 3.2). Spectra and associated instrument response files were then extracted for each observation using the CIAO tool psextract using a circular extraction

region of radius $\mathrm{R}_{e x t}=2$. . $^{\prime} 5$ centered on the X-ray source. Background was extracted from adjacent source-free regions on the same CCD and is negligible, comprising only about $0.7 \%$ of the total counts within the source extraction region. Spectra were rebinned to a minimum of 20 counts per bin and analyzed using XSPEC vers. 12.2.0bp. We integrated the magnetar atmosphere model described by P01 into XSPEC for spectral fitting.

\section{Pulsar X-ray Properties}

The pulsar was identified on the basis of $10.61 \mathrm{~s}$ pulsations discovered in the brightest X-ray source detected in the Chandra Wd 1 observations, lying $\approx 1.6^{\prime}$ southeast of the $\mathrm{Wd}$ 1 core (Fig. 1 of S06; Fig. 2 of M06). Timing analysis was discussed by M06 and will not be discussed further in this paper. Higher time resolution observations will be needed to characterize the pulse properties.

The Chandra position of the pulsar as determined by the CIAO wavdetect wavelet detection tool using data from the longer exposure on 18-19 June 2005 is (J2000.0) R.A. $=16^{h}$ $47^{m} 10 .{ }^{s} 20$, decl. $=-45^{\circ} 52^{\prime} 17.05^{\prime \prime}$. The $90 \%$ source location error circle for ACIS-S ${ }^{2}$ has a radius of $\approx 0 . .^{\prime \prime} 5$. The X-ray centroid obtained from the XIMAGE image analysis software package is nearly identical. The May 2005 observation detected $386 \pm 20$ net counts and the June observation yielded $834 \pm 29$ net counts, based on events in the $0.3-7 \mathrm{keV}$ range. The respective count rates were $2.05( \pm 0.11) \times 10^{-2} \mathrm{c} \mathrm{s}^{-1}$ and $2.17( \pm 0.08) \times 10^{-2} \mathrm{c} \mathrm{s}^{-1}$, which are the same to within the uncertainties. Photon pileup is negligible $(\leq 3 \%)$. Since no optical or IR counterpart has yet been found, cluster membership has not been proven and

\footnotetext{
${ }^{1}$ Further information on Chandra Interactive Analysis of Observations (CIAO) software can be found at http://asc.harvard.edu/ciao.

${ }^{2}$ Chandra Proposer's Observatory Guide, Rev. 8.0, Dec. 2005; http://cxc.harvard.edu
} 
the distance is uncertain. Spectroscopic studies give a distance to $\mathrm{Wd} 1$ in the range $\mathrm{d}=2$ - $5.5 \mathrm{kpc}$ (Clark et al. $2005=\mathrm{C} 05)$ and the photometric study of Brandner et al. $(2005=$ B05) gives $\mathrm{d}=4 \mathrm{kpc}$. In the following we adopt a distance $\mathrm{d}=5 \mathrm{kpc}$ to the pulsar based on the assumption that it is associated with $\mathrm{Wd} 1$.

Figures 1 and 2 show the ACIS-S spectrum of the pulsar. The spectrum is heavily absorbed below $\approx 1 \mathrm{keV}$. Strong absorption is anticipated if the pulsar lies in (or behind) Wd 1 based on cluster extinction estimates and the total galactic HI column density toward the pulsar, which is $\mathrm{N}_{\mathrm{H}}=2.2 \times 10^{22} \mathrm{~cm}^{-2}$ based on the HEASARC $\mathrm{N}_{\mathrm{H}}$ calculation tool

3. Cluster extinction estimates are in the range $A_{V} \approx 9.5-13.6$ mag (B05; C05) which corresponds to $\mathrm{N}_{\mathrm{H}} \approx(2.1-3.0) \times 10^{22} \mathrm{~cm}^{-2}$ using the conversion of Gorenstein (1975). Our spectral fits of the Wd 1 pulsar with models that include a thermal (e.g. blackbody) component yield $\mathrm{N}_{\mathrm{H}}$ estimates that are at or slightly below the low end of this range (Table 1).

The pulsar spectrum shows no obvious lines with the possible exception of a weak emission feature near $3.5 \mathrm{keV}$. As Figure 3 shows, this feature is most apparent in the longer second observation but is only weakly present (if at all) in the shorter first observation. Since the feature is not clearly present in the first observation, its reality is in doubt. There are no known thermal emission lines near $\mathrm{kT}=3.5 \mathrm{keV}$ or near $\mathrm{kT}=4.5 \mathrm{keV}$, where the latter value is corrected for gravitational redshift $\left(\mathrm{M}_{n s}=1.4 \mathrm{M}_{\odot}, \mathrm{R}_{n s}=10 \mathrm{~km}\right)$. We are not aware of any instrumental effects that would give rise to a feature near $3.5 \mathrm{keV}$. There is a gold $\mathrm{M}$ edge at $3.428 \mathrm{keV}$ but a detectable emission feature from this edge seems unlikely. If the feature is confirmed as an emission line in higher signal-to-noise spectra, a nonthermal interpretation will likely be required.

\section{X-ray Spectral Models}

For the spectral analysis discussed below, we focus on the spectrum from the second observation in June 2005, which provides the best signal-to-noise ratio. We attempted to fit the spectrum with a variety of emission models, as summarized in Table 1. All models included an absorption component based on cross-sections from Morrison \& McCammon (1983).

\footnotetext{
${ }^{3}$ http://heasarc.gsfc.nasa.gov/cgi-bin/Tools/w3nh/w3nh.pl
} 


\subsection{Power Law Model}

Before considering models that include a thermal emission component, we note for completeness that the spectrum can be acceptably fitted with a simple power-law model (model A in Table 1). The $\mathrm{N}_{\mathrm{H}}$ and unabsorbed X-ray luminosity $\log \mathrm{L}_{X}=34.44 \mathrm{ergs} \mathrm{s}^{-1}$ determined from this power-law model are larger than obtained from models that include thermal emission and the inferred $\mathrm{N}_{\mathrm{H}}$ is at or above the maximum expected from $\mathrm{A}_{\mathrm{V}}$ estimates (Sec. 3). Simple power-law models have been used to fit the soft-band X-ray spectra of some rotation-powered pulsars (Becker \& Trümper 1997), but acceptable fits of AXPs based on good-quality X-ray spectra typically require at least two emission components (e.g. blackbody + power-law). Thus, if the Wd 1 pulsar is an AXP then the ability to fit its spectrum with a simple power-law model may be a consequence of limited signal-to-noise in the existing Chandra data. The rather high $\mathrm{N}_{\mathrm{H}}$ deduced from the power-law model motivates us to consider models that include a thermal component, as discussed below.

\subsection{Blackbody Models}

An isothermal blackbody model (model B in Table 1) yields an acceptable fit with $\mathrm{kT}_{b b}=0.59 \mathrm{keV}$. Although the fit is formally acceptable $\left(\chi^{2} /\right.$ dof $\left.=34.7 / 35=0.99\right)$, X-ray modeling of other AXPs (P01) suggests that a power-law component may also be present. A two-component blackbody + power-law model (model $\mathrm{C}$ in Table 1) provides only a minor improvement in the $\chi^{2}$ fit statistic. Thus, the existing Chandra data are consistent with a two-component blackbody + power-law model but it is not necessary to include both components to obtain an acceptable fit. Our best-fit blackbody + power-law model gives a photon power-law index $\alpha_{p h} \approx 1.8$, but this value is not tightly constrained. In this blackbody + power-law model the power-law component could contribute as much as $\approx 40 \%$ of the observed (absorbed) flux in the $0.3-8 \mathrm{keV}$ range (Table 1 ).

The inferred source radius is in the range $R_{s}=0.27-0.36 \mathrm{~km}$ for the two blackbody models in Table 1. These values are much less than the radius of a neutron star. It is thus obvious that we are not detecting thermal emission emanating from the surface of a neutron star, assuming the intrinsic spectrum is an isothermal blackbody. As already noted (Sec. 1), blackbody models may yield incorrect radii due to atmospheric effects. We investigate this possibility below in the case where the X-ray source is assumed to be a magnetar. 


\subsection{Magnetar Models}

The magnetar model developed by P01 assumes an underlying highly magnetized neutron star of mass $\mathrm{M}_{n s}=1.4 \mathrm{M}_{\odot}$ cooling via blackbody emission that is reprocessed through a light-element highly magnetized atmosphere (Heyl \& Hernquist 1998). The model computes the phase-averaged flux as a function of photon energy $E$, taking into account anisotropic heat flow through the envelope due to magnetic field effects and general relativistic light deflection (eqs. 3-5 of P01). The phase-averaged magnetar flux $F(E)$ at a distance $d$ from the star to observer without any correction for absorption by intervening material is (eq. [3] of P01 in corrected form):

$$
F(E)=\frac{R_{\infty}^{2} \sigma T_{p, \infty}^{4}}{d^{2}} \frac{1}{k T_{p, \infty}} \times \int_{0}^{2 \pi} \frac{d \alpha}{2 \pi} \int_{0}^{1} 2 x d x \int_{0}^{2 \pi} \frac{d \phi}{2 \pi} I_{0}(\theta, \phi) n\left(E e^{-\xi}, T_{s}(\theta, \phi)\right)
$$

In the above, $T_{p, \infty} \equiv \mathrm{T}_{p} e^{\xi}$ where $\mathrm{T}_{p}$ is the pole temperature, $R_{\infty} \equiv \mathrm{R}_{n s} e^{-\xi}$, and $e^{\xi}$ $\equiv \sqrt{1-\left(R_{s c h} / R_{n s}\right)}$ where $R_{n s}$ is the stellar radius, $R_{s c h}=2 \mathrm{GM}_{n s} / \mathrm{c}^{2}$ is the Schwarzschild radius, and the integrated spectrum over the stellar surface accounts for general relativistic light deflection and the effects of a light-element atmosphere. The angle $\alpha$ is the angle between the magnetic pole and line-of-sight, $\delta$ is the angle between an emitted photon and the normal to the surface, and $x \equiv \sin \delta$. The spherical coordinate angles $(\theta, \phi)$ specify the position on the surface of the star, $I_{0}(\theta, \phi)$ is the surface intensity distribution (eq. [5] of $\mathrm{P} 01)$, and $n\left(E e^{-\xi}, T_{s}(\theta, \phi)\right)$ specifies the local emission at a point on the surface as a function of the local temperature $T_{s}$ (eq. [6] of P01).

We incorporated the magnetar model of P01 into XSPEC and reran the blackbody fits discussed above, replacing the blackbody model with the magnetar model. The magnetar fit results are summarized as models $\mathrm{D}$ and $\mathrm{E}$ in Table 1 . In our implementation, the radius of the neutron star and the stellar distance were specified as input parameters. For the fits in Table 1 we assumed $\mathrm{R}_{n s}=10 \mathrm{~km}$ and $\mathrm{d}=5 \mathrm{kpc}$. The model was used to find the best-fit value of the neutron star pole temperature and flux normalization factor norm $_{1}=$ $\mathrm{F}_{X, t h} / \mathrm{F}_{X, m a g}$, where $\mathrm{F}_{X, t h}$ is the unabsorbed flux due to the thermal component over the fitted energy range and $\mathrm{F}_{X, m a g}$ is the flux predicted by the model for a magnetar of the assumed radius and distance.

As Table 1 shows, the $\chi^{2}$ fit statistics for the magnetar models are comparable to or slightly better than those for the blackbody models. The overall fit for the two-component magnetar + power-law model (model E) shown in Figure 4 looks nearly identical to that of the blackbody + power-law model (Fig. 1). However, a comparison of the unfolded spectra

in Figures 2 and 5 shows that contribution of a power-law component is much less in the 
magnetar + power-law model. The overall shape of the spectrum is matched quite well with the magnetar component alone, and any power-law component need not contribute more than $\approx 15 \%$ of the observed flux $(0.3-8 \mathrm{keV})$.

The inferred magnetar pole temperatures are in the range $\mathrm{kT}_{p}=0.44-0.48 \mathrm{keV}$. These values are $10 \%$ - 20\% less than those obtained with the corresponding blackbody models, but still too high to be reconciled with thermal cooling alone. A similar result was noted by P01 when fitting $A S C A$ spectra for five AXPs, and they concluded that additional heating (possibly by magnetic field decay) is needed to explain the high temperatures.

It is apparent from Table 1 that the flux normalization factor norm ${ }_{1}$ is much less than unity for the magnetar models. By definition of norm $_{1}$ (see above), this indicates that the unabsorbed flux predicted by the magnetar model with $\mathrm{R}_{n s}=10 \mathrm{~km}$ and $\mathrm{d}=5 \mathrm{kpc}$ is $\sim 10^{2}$ greater than that determined from the X-ray spectrum. Since the leading term in the magnetar flux relation (eq. 1) is the blackbody flux, the flux mismatch indicates that the source radius is much less than $\mathrm{R}_{n s}$, provided that $\mathrm{d}=5 \mathrm{kpc}$ is not a serious underestimate. This is shown in the last row of Table 1 , which gives the source radius $\mathrm{R}_{s}$ determined from the blackbody formula (which we emphasize is only an approximation in the magnetar case). The discrepancy could be removed by assuming the pulsar is $\sim 10$ times more distant, but this seems unlikely if the pulsar is indeed associated with $\mathrm{Wd} 1$. We thus conclude that the observed X-ray emission cannot be readily explained as global emission coming from the surface of a magnetar cooling through a light-element atmosphere.

\section{Discussion}

The spectral analysis in Section 4 shows the following: (i) most of the observed Xray flux in the Chandra bandpass can be accounted for by thermal models but a possible power-law contribution is not ruled out by the existing data, (ii) thermal models give a characteristic temperature in the range $\mathrm{kT} \approx 0.4-0.6 \mathrm{keV}$, which is higher than expected for neutron star cooling alone, (iii) the inferred source radius from blackbody models is $\mathrm{R}_{s}$ $<0.4 \mathrm{~km}$ and (iv) a magnetar model for a neutron star of radius $\mathrm{R}_{n s}=10 \mathrm{~km}$ emitting from its entire surface overestimates the flux, indicative of an emitting region that is considerably smaller than $\mathrm{R}_{n s}$ unless the pulsar distance $\mathrm{d}=5 \mathrm{kpc}$ is significantly underestimated.

Thus, the models considered here point to a small emitting region of relatively warm plasma ( $\mathrm{T} \sim 5-7 \mathrm{MK}$ ) as the origin of most of the observed X-ray emission. A plausible explanation is that the emission arises from one or more high-temperature regions or "hot spots" on the neutron star surface, rather than from the entire surface of a cooling neutron 
star. We obtain an equivalent blackbody source radius in the range $R_{s}=0.27-0.52 \mathrm{~km}$ (Table 1), but this does not necessarily correspond to the radius of any particular spot since multiple spots may be present. We have assumed $\mathrm{d}=5 \mathrm{kpc}$ but the conclusion of a small emitting region $\mathrm{R}_{s}<<\mathrm{R}_{n s}$ holds even if the distance is twice that value. The ratio of source emitting area to stellar surface area $\left(\mathrm{R}_{s} / \mathrm{R}_{n s}\right)^{2} \sim 10^{-3}$ obtained here is comparable to that obtained for some field pulsars with magnetic field strengths below the magnetar range (e.g. Greiveldlinger et al. 1996) but is smaller than the typical ratio $\left(\mathrm{R}_{s} / \mathrm{R}_{n s}\right)^{2} \sim 10^{-1}$ derived for the AXPs studied by Durant \& van Kerkwijk (2006).

Spots can form on a magnetar as a result of the local concentration of magnetic field lines (TD96). In that case, the equivalent blackbody temperature of a spot is expected to anti-correlate with the spot radius for objects having the same $\mathrm{L}_{X}$ (eq. [92] of TD96). The calculation of equivalent blackbody radii made possible by new distance determinations of several galactic AXPs (Durant \& van Kerkwijk 2006) suggests that such an anti-correlation may be present.

Spots can also form as a result of accretion onto the neutron star, even if it is not a magnetar. Assuming that the Wd 1 pulsar does not have a low-mass companion, the accretion reservoir could in principle be a fallback disk created after the supernova explosion (Chatterjee et al. 2000; Alpar 2001) or the ISM (Blaes \& Madau 1993). More sensitive images in the infrared and at millimeter wavelengths are needed to determine if a disk is present. This question is of considerable interest given the recent detection of mid-IR emission at the position of the isolated young pulsar $4 \mathrm{U} 0142+61$ (Wang et al. 2006). These authors argue that the mid-IR emission arises in a passive X-ray heated disk around the neutron star that may have originated from supernova ejecta that subsequently fell back onto the neutron star.

Until observational evidence for a disk around the Wd 1 pulsar is presented, any conclusions based on disk models should be considered speculative. We only remark that if the pulsation period $\mathrm{P}=10.6 \mathrm{~s}$ is close to the equilibrium period for uniform spin-down by a disk (eq. [6] of TD96) then a dipole field strength $\mathrm{B}_{\text {dipole }} \sim 10^{11} \mathrm{G}$. is inferred from $\mathrm{L}_{X}$ (Table 1). This field strength is a lower limit if the detected emission is dominated by spots since the total $\mathrm{L}_{X}$ would contain an additional contribution from the cooling surface of the neutron star that may be masked by absorption. Accretion from the ISM onto polar caps could achieve temperatures $\mathrm{kT}_{b b} \approx 0.5 \mathrm{keV}$ (Table 1) for realistic accretion rates (eq. [29] of Blaes \& Madau 1993). Even so, the accretion rate and gas density around the source needed to account for $\mathrm{L}_{X} \sim 10^{33} \mathrm{ergs} \mathrm{s}^{-1}$ (Table 1) are uncomfortably high and the ISM accretion picture is difficult to justify.

If the observed emission is coming predominantly from one or more hot spots, then where is the global emission from the neutron star surface? A likely explanation is that the 
surface emission is largely absorbed due to the relatively high intervening absorption column toward the pulsar. If we assume a soft intrinsic blackbody spectrum with $\mathrm{kT}_{b b}=0.1 \mathrm{keV}$ for a cooling neutron star with $\mathrm{R}_{n s}=10 \mathrm{~km}$ then the unabsorbed broadband flux at $\mathrm{d}=5 \mathrm{kpc}$ is $\mathrm{F}_{X}=4.3 \times 10^{-13} \mathrm{ergs} \mathrm{cm}^{-2} \mathrm{~s}^{-1}$. Assuming $\mathrm{N}_{\mathrm{H}}=1.7 \times 10^{22} \mathrm{~cm}^{-2}$ (a typical value from Table 1 ), the PIMMS ${ }^{4}$ simulator predicts that no more that $\approx 5$ counts would have been detected in the second Chandra ACIS-S exposure $(38.5 \mathrm{ksec})$ from this hypothetical blackbody. If the neutron star is a magnetar, then theoretical cooling curves (Heyl \& Kulkarni 1998) predict $\mathrm{kT} \leq 0.1 \mathrm{keV}$ for ages $t \gtrsim 0.2 \operatorname{Myr}\left(\mathrm{B} \sim 10^{14} \mathrm{G}\right)$.

Finally, we comment on the X-ray luminosity of the $\mathrm{Wd} 1$ pulsar and its relevance to the AXP classification and the energy source that powers the X-ray emission. The maximum $\mathrm{X}$-ray luminosity determined from models that include a thermal component is $\log \mathrm{L}_{X}=$ $33.20 \operatorname{ergs~s}^{-1}$ (model $\mathrm{C}$ in Table 1 ). This $\mathrm{L}_{X}$ along with the inferred blackbody temperature $\mathrm{kT}_{b b} \approx 0.5 \mathrm{keV}$ places the $\mathrm{Wd} 1$ pulsar in the $\left(\mathrm{kT}_{b b}, \mathrm{~L}_{X}\right)$ plane at a temperature similar to other known AXPs but at a luminosity that is at least 10 times lower (Fig. 5 of M02). This conclusion is strengthened by the recent work of Durant \& van Kerkwijk (2006), who conclude that AXP X-ray luminosities are typically $\mathrm{L}_{X} \sim 10^{35} \mathrm{ergs} \mathrm{s}^{-1}$. It thus appears that the Wd 1 pulsar is underluminous in X-rays relative to previously known AXPs.

There are several possible explanations for the apparent low X-ray luminosity, apart from the obvious possibility of an underestimated distance. Since the X-ray temperature obtained for the Wd 1 pulsar is similar to other AXPs (P01) the lower $\mathrm{L}_{X}$ may just be an indication of a smaller emitting area (i.e. emission dominated by highly localized spots). Alternatively, if the Wd 1 pulsar and other AXPs are magnetars powered by magnetic field decay, then the lower $\mathrm{L}_{X}$ for the $\mathrm{Wd} 1$ pulsar could be an indication of lower magnetic field strength (B). The surface heat flux of a magnetar scales sensitively as $\mathrm{B}^{4.4}$ (eq. [91] of TD96). Although $\mathrm{L}_{X}$ for the Wd 1 pulsar does seem low, it should be kept in mind that only a few AXPs are presently known and the AXP X-ray luminosity function is not well-sampled. Observational selection effects may have biased previous AXP identifications toward more X-ray luminous members of the class. Finally, the discrepancy could be resolved if the simple power-law model (model A) is correct. However, as we have already noted, a simple power-law model is likely an over-simplification of the true intrinsic spectrum if the Wd 1 pulsar is an AXP.

Do we need to invoke the magnetar interpretation for the Wd 1 pulsar? Could its X-ray luminosity be powered by spin-down instead of an ultra-strong magnetic field? The maximum X-ray luminosity that can be derived from rotational energy release for a pulsar

\footnotetext{
${ }^{4}$ http://asc.harvard.edu/toolkit/pimms.jsp
} 
of period $\mathrm{P}$, age $t$, and moment of inertia $I$ is (eq. [2] of TD96)

$$
L_{X} \sim \frac{1}{2 t} I\left[\frac{2 \pi}{\mathrm{P}}\right]^{2} \operatorname{ergs~\mathrm {s}^{-1}} .
$$

Using $\mathrm{P}=10.6 \mathrm{~s}$ and assuming $\mathrm{M}_{n s}=1.4 \mathrm{M}_{\odot}$ and $\mathrm{R}_{n s}=10 \mathrm{~km}$, the above becomes

$$
t_{4} \sim \frac{6 \times 10^{32}}{\mathrm{~L}_{\mathrm{X}}\left(\operatorname{ergs~s}^{-1}\right)}
$$

where $t_{4}$ is the age in units of $10^{4} \mathrm{yr}$. From the thermal-component models in Table 1 we have $\mathrm{L}_{X} \approx 1.4 \times 10^{33} \mathrm{ergs} \mathrm{s}^{-1}$, but as noted above this $\mathrm{L}_{X}$ should be considered a lower limit on the total X-ray luminosity (neutron star cooling surface + hot spots). In that case, spin-down is a plausible energy source if the $\mathrm{Wd} 1$ pulsar age is $t \lesssim 4 \times 10^{3} \mathrm{yr}$. The two Chandra observations give an upper limit on the period derivative $\dot{\mathrm{P}}<2 \times 10^{-10} \mathrm{~s} \mathrm{~s}^{-1}$ (M06). Using the relation $\dot{\mathrm{E}}=-4 \pi^{2} I \dot{\mathrm{P}} / \mathrm{P}^{3}$ we obtain an an upper limit on the rate of energy release from spin-down of $\log \dot{\mathrm{E}}<33.87 \mathrm{ergs} \mathrm{s}^{-1}$. As can be seen (Table 1), the X-ray luminosities inferred from thermal-component spectral fits are a few times less than the above value of E.

\section{Summary}

The X-ray emission detected by Chandra from the $10.6 \mathrm{~s}$ pulsar in Wd 1 is not global surface emission from a cooling neutron star or magnetar. Both blackbody and magnetar models imply an emitting region that is much smaller than the radius of a neutron star. Models that include a thermal component show that the observed emission most likely arises from one or more hot spots $(\mathrm{kT} \approx 0.5 \mathrm{keV})$ covering a small fraction of the surface. Any cool $(\mathrm{kT} \leq 0.1 \mathrm{keV})$ emission from the cooling surface would have been heavily attenuated by intervening absorption.

The Wd 1 pulsar shows X-ray properties that justify its tentative classification as an AXP. Even so, the X-ray luminosity $\mathrm{L}_{X} \approx 10^{33.15}(\mathrm{~d} / 5 \mathrm{kpc})^{2} \operatorname{ergs~s}^{-1}(0.3-8 \mathrm{keV})$ of the pulsar deduced from thermal spectral models is at least an order of magnitude below that

of known AXPs if $\mathrm{d} \approx 5 \mathrm{kpc}$. The existing constraint on $\dot{\mathrm{P}}$ from two Chandra observations is not stringent enough to rule out spin-down as the energy source if the pulsar is young and if its X-ray luminosity is indeed as low as $\mathrm{L}_{X} \approx 10^{33.15} \mathrm{ergs} \mathrm{s}^{-1}$.

Since the pulsar has only recently been discovered, further observational work will be 
needed to clarify its nature. Deeper optical/IR/millimeter searches for a low-mass companion or residual disk will be particularly important for the X-ray interpretation. A higher signalto-noise X-ray spectrum is needed to distinguish between competing emission models and a tighter constraint on $\dot{\mathrm{P}}$ from continued X-ray time monitoring would be useful to determine if spin-down can account for the X-ray luminosity.

This research was supported by NASA grant GO5-6009X. 


\section{REFERENCES}

Alpar, M.A., 2001, ApJ, 554, 1245

Blaes, O. \& Madau, P., 1993, ApJ, 403, 690

Becker, W. \& Trümper, J., 1997, A\&A, 326, 682

Brandner, W., Clark, S., \& Waters, R., 2005, in Protostars and Planets V, contribution no. 1286, p. 8344 , http://www.lpi.usra.edu/meetings/ppv2005/ (B05)

Chatterjee, P., Hernquist, L., \& Narayan, R., 2000, ApJ, 534, 373

Clark, J.S., Negueruela, I., Crowther, P.A., \& Goodwin, S.P., 2005, A\&A, 434, 949 (C05)

Duncan, R.C. \& Thompson, C., 1992, ApJ, 392, L9

Durant, M. \& van Kerkwijk, M.H., 2006, ApJ, in press (astro-ph/0606027)

Gorenstein, P., 1975, ApJ, 198, 95

Gotthelf, E.V. \& Halpern, J.P., 2005, ApJ, 632, 1075 (GH05)

Greiveldinger, C. et al., 1996, ApJ, 465, L35

Heyl, J.S. \& Hernquist, L., 1998, MNRAS, 298, L17

Heyl, J.S. \& Kulkarni, S.R., 1998, ApJ, 506, L61

Kaspi, V.M. \& Gavriil, F.P., 2004, Nuc. Pys. B (Proc. Suppl.), 132, 456

Mereghetti, S., Chiarlone, L., Israel, G.L., \& Stella, L., 2002, in Neutron Stars, Pulsars, and Supernova Remnants, eds. W. Becker, H. Lesch, \& J. Trümper, MPR Report 278, 29

Morrison, R. \& McCammon, D., 1983, ApJ, 270, 119

Muno, M.P. et al., 2006, ApJ, 636, L41 (M06)

Perna, R., Heyl, J.S., Hernquist, L.E., Juett, A.M., \& Chakrabarty, D., 2001, ApJ, 557, 18 (P01)

Skinner, S.L., Damineli, A., Palla, F., Zhekov, S.A., Simmons, A.E., \& Teodoro, M., 2005, BAAS, 37 (4), 1279 (S05)

Skinner, S.L., Simmons, A.E., Zhekov, S.A., Teodoro, M., Damineli, A., \& Palla, F., 2006, ApJ, 638, L35 (S06) 
Thompson, C. \& Duncan, R.C., 1996, ApJ, 473, 322 (TD96)

van Paradijs, J., Taam, R.E., \& van den Heuvel, E.P.J., 1995, A\&A, 299, L41

Wang, Z., Chakrabarty, D., \& Kaplan, D.L., 2006, Nature, 440, 772 
Table 1. Chandra ACIS-S Spectral Fits for the Wd 1 Pulsar

\begin{tabular}{|c|c|c|c|c|c|}
\hline \multicolumn{6}{|l|}{ Parameter } \\
\hline Model $^{\mathrm{a}}$ & $\mathrm{A}$ & B & $\mathrm{C}$ & $\mathrm{D}$ & $\mathrm{E}$ \\
\hline Components & $\mathrm{pl}$ & $\mathrm{bb}$ & $\mathrm{bb}+\mathrm{pl}$ & mag & $\mathrm{mag}+\mathrm{pl}$ \\
\hline $\mathrm{N}_{\mathrm{H}}\left(10^{22} \mathrm{~cm}^{-2}\right)$ & $3.1[2.7-3.6]$ & $1.5[1.2-1.8]$ & $1.8[1.3-3.2]$ & $1.7[1.4-2.0]$ & $1.8[1.4-3.9]$ \\
\hline $\mathrm{kT}(\mathrm{keV})$ & $\ldots$ & $0.59[0.54-0.63]$ & $0.50[0.36-0.60]$ & $0.48[0.42-0.53]$ & $0.44[0.31-0.52]$ \\
\hline $\operatorname{norm}_{1} \mathrm{~b}$ & $\ldots$ & 0.30 & 0.53 & $4.6 \mathrm{e}-03$ & $6.1 \mathrm{e}-03$ \\
\hline$\alpha_{p h}$ & $3.8[3.4-4.2]$ & $\ldots$ & $1.8[\ldots]$ & $\ldots$ & $1.8[\ldots]$ \\
\hline $\operatorname{norm}_{p l}$ & $1.2 \mathrm{e}-03$ & $\ldots$ & $3.1 \mathrm{e}-05$ & $\ldots$ & $1.2 \mathrm{e}-05$ \\
\hline$\chi^{2} /$ dof & $35.3 / 35$ & $34.7 / 35$ & $32.0 / 33$ & $32.2 / 35$ & $31.8 / 33$ \\
\hline$\chi_{r e d}^{2}$ & 1.0 & 0.99 & 0.97 & 0.92 & 0.96 \\
\hline $\mathrm{F}_{\mathrm{X}}^{\mathrm{c}}$ & $2.14(91.9)$ & $1.93(3.79)$ & $2.18(5.37)$ & $2.00(4.37)$ & $2.09(5.03)$ \\
\hline $\mathrm{F}_{\mathrm{X}, \mathrm{th}}{ }^{\mathrm{c}}$ & $\ldots$ & $1.93(3.79)$ & $1.34(3.56)$ & $2.00(4.37)$ & $1.76(4.32)$ \\
\hline $\log L_{X}\left(\operatorname{ergs~s}{ }^{-1}\right)$ & 34.44 & 33.05 & 33.20 & 33.12 & 33.18 \\
\hline $\mathrm{R}_{s}(\mathrm{~km})$ & $\ldots$ & 0.27 & 0.36 & 0.44 & 0.52 \\
\hline
\end{tabular}

Note. - Based on XSPEC (vers. 12.2.0) fits of the background-subtracted ACIS-S spectrum of the Wd 1 pulsar (CXO J164710.20-455217) binned to 20 counts per bin using 38,473 s ksec of exposure obtained on 18-19 June 2005. Blackbody (bb) emission was modeled with the bbodyrad model in XSPEC. Magnetar (mag) emission was modeled with the custom model magnetar (see text). The tabulated parameters are absorption column density $\left(\mathrm{N}_{\mathrm{H}}\right)$, blackbody temperature or magnetar pole temperature $(\mathrm{kT})$, normalization of the blackbody or magnetar component $\left(\right.$ norm $\left._{1}\right)$, photon power-law index $\left(\alpha_{p h}\right)$, power-law normalization $\left(\right.$ norm $\left._{p l}\right)$. Square brackets enclose $90 \%$ confidence intervals and an ellipsis means that the algorithm used to compute confidence intervals did not converge. The total X-ray flux $\left(\mathrm{F}_{\mathrm{X}}\right)$ is the absorbed value in the $0.3-8 \mathrm{keV}$ range, followed in parentheses by unabsorbed value. The thermal flux associated with the blackbody or magnetar component is $\mathrm{F}_{\mathrm{X}, \mathrm{th}}$. The unabsorbed luminosity $\mathrm{L}_{\mathrm{X}}(0.3-8 \mathrm{keV})$ assumes a distance of $5 \mathrm{kpc} . \mathrm{R}_{s}$ is the inferred blackbody source radius at $\mathrm{d}=5 \mathrm{kpc}$ based on the relation $\mathrm{R}_{s}^{2}=\mathrm{L}_{X, t h} /\left(4 \pi \sigma \mathrm{T}^{4}\right)$ where $\mathrm{L}_{X, t h}$ is the luminosity associated with the unabsorbed thermal flux $\mathrm{F}_{X, t h}$. This relation is only approximate for models $\mathrm{D}$ and $\mathrm{E}$ since magnetar spectra are not true blackbodies.

${ }^{\text {a }}$ Model A: $\mathrm{N}_{\mathrm{H}} \cdot(\mathrm{PL})$; Model B: $\mathrm{N}_{\mathrm{H}} \cdot\left(\mathrm{kT}_{b b}\right)$; Model C: $\mathrm{N}_{\mathrm{H}} \cdot\left(\mathrm{kT}_{b b}+\mathrm{PL}\right)$; Model $\mathrm{D}: \mathrm{N}_{\mathrm{H}} \cdot\left(\mathrm{kT}_{\text {magnetar }}\right)$; Model $\mathrm{E}: \mathrm{N}_{\mathrm{H}} \cdot\left(\mathrm{kT}_{\text {magnetar }}+\mathrm{PL}\right)$

${ }^{\mathrm{b}}$ For models $\mathrm{B}$ and $\mathrm{C}$ : norm $1=\mathrm{R}_{k m}^{2} / \mathrm{d}_{10}^{2}$ where $\mathrm{R}_{k m}$ is the source radius in $\mathrm{km}$ and $\mathrm{d}_{10}$ is the distance to the source in units of $10 \mathrm{kpc}$. For models $\mathrm{D}$ and $\mathrm{E}$ : norm $\mathrm{n}_{1}=\mathrm{F}_{X, t h} / \mathrm{F}_{\text {mag }}$ where $\mathrm{F}_{X, t h}$ is the unabsorbed thermal flux flux determined from the spectrum and $\mathrm{F}_{\text {mag }}$ is the theoretical flux predicted for a magnetar with radius $\mathrm{R}=10 \mathrm{~km}$ and distance $\mathrm{d}=5 \mathrm{kpc}$ using the model of P01.

${ }^{\mathrm{c}}$ Flux units are $10^{-13} \mathrm{ergs}_{\mathrm{cm}}^{-2} \mathrm{~s}^{-1}$. 


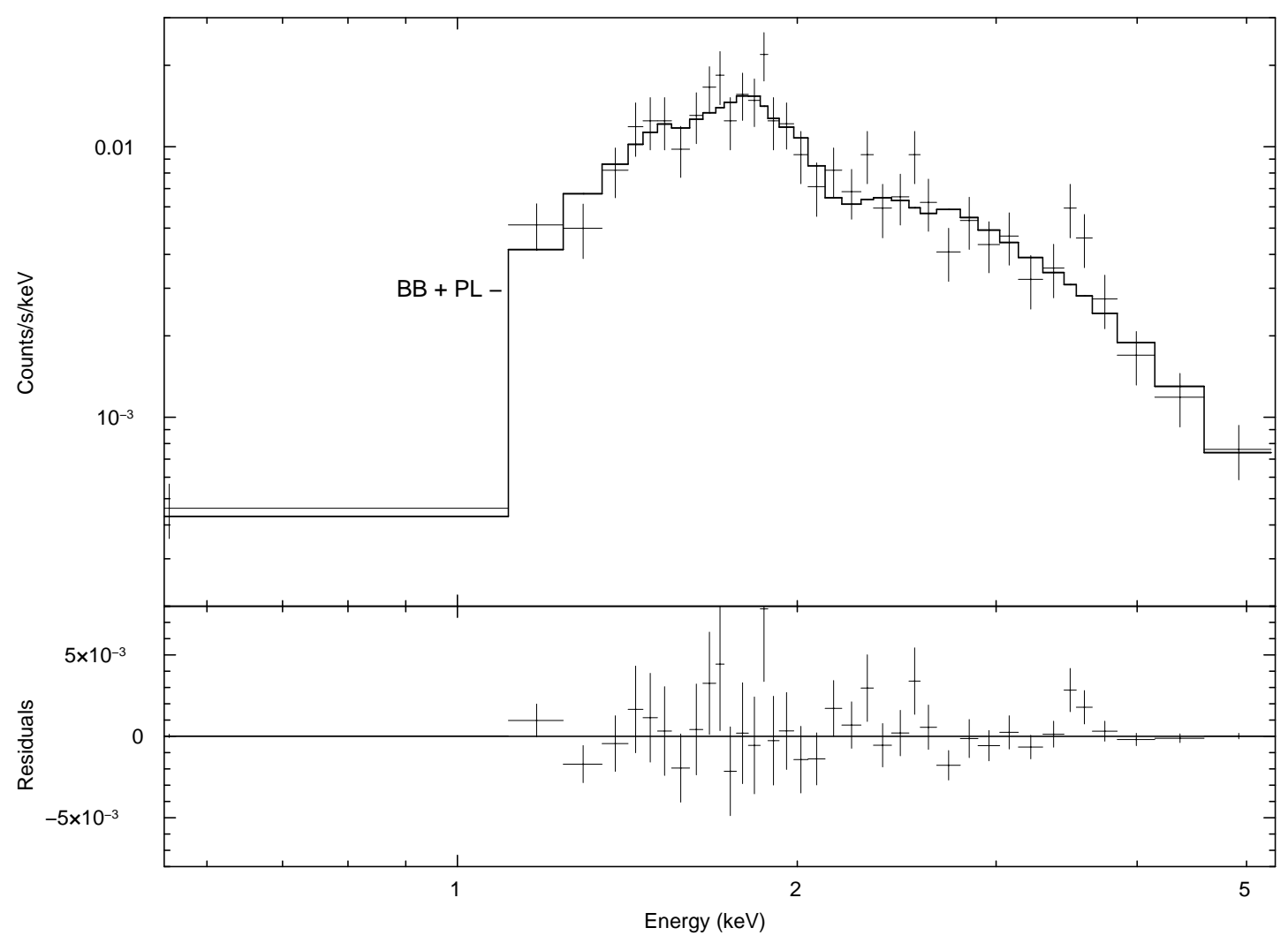

Fig. 1.- Background-subtracted Chandra ACIS-S spectrum of the pulsar CXO J164710.20-455217 obtained on 18-19 June 2005 (834 counts). The spectrum is rebinned to a minimum of 20 counts per bin. The overlaid model is a 2-component blackbody + power-law (model C in Table 1). 


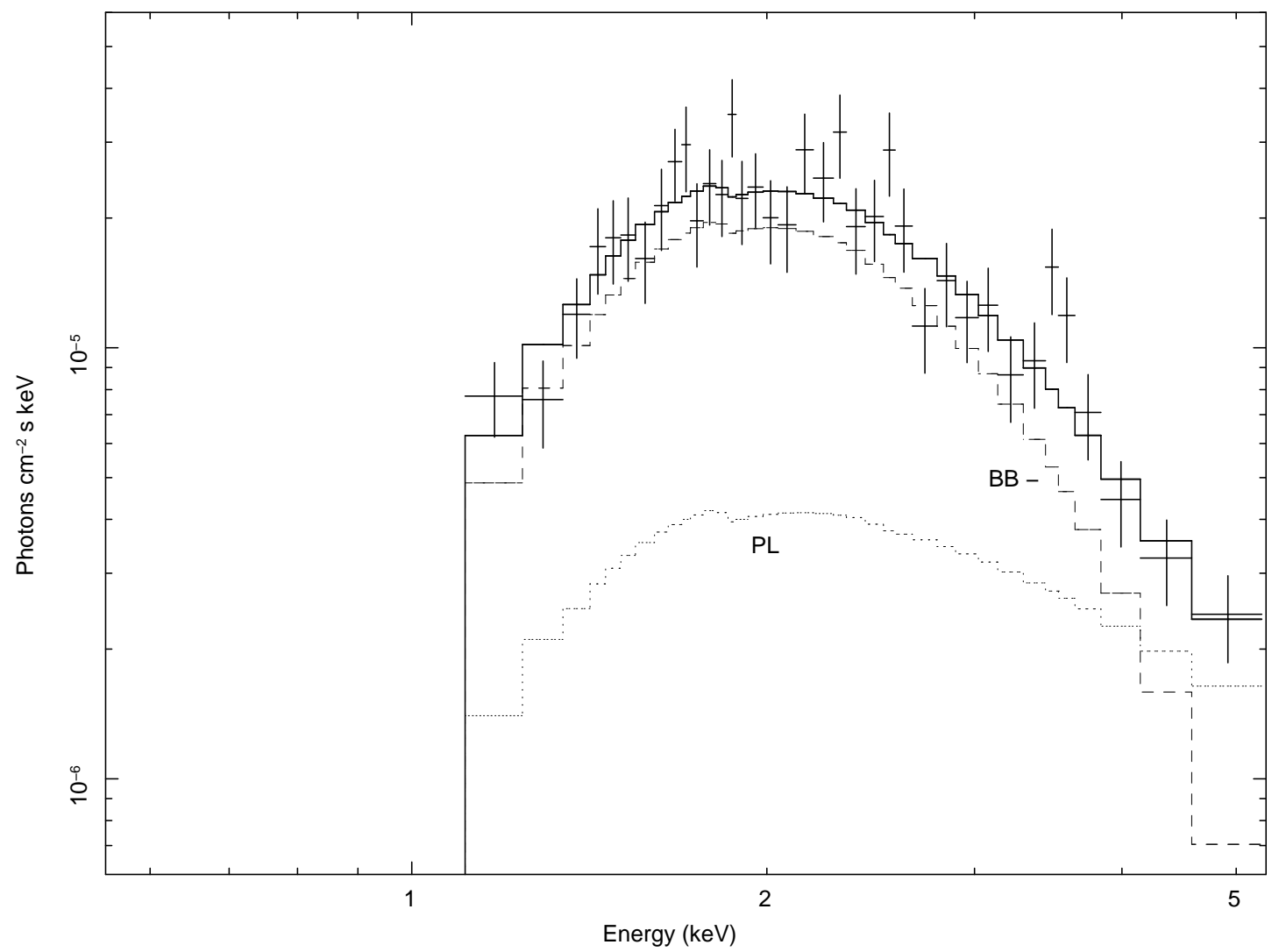

Fig. 2.- Same as Figure 1 showing the unfolded spectrum. 


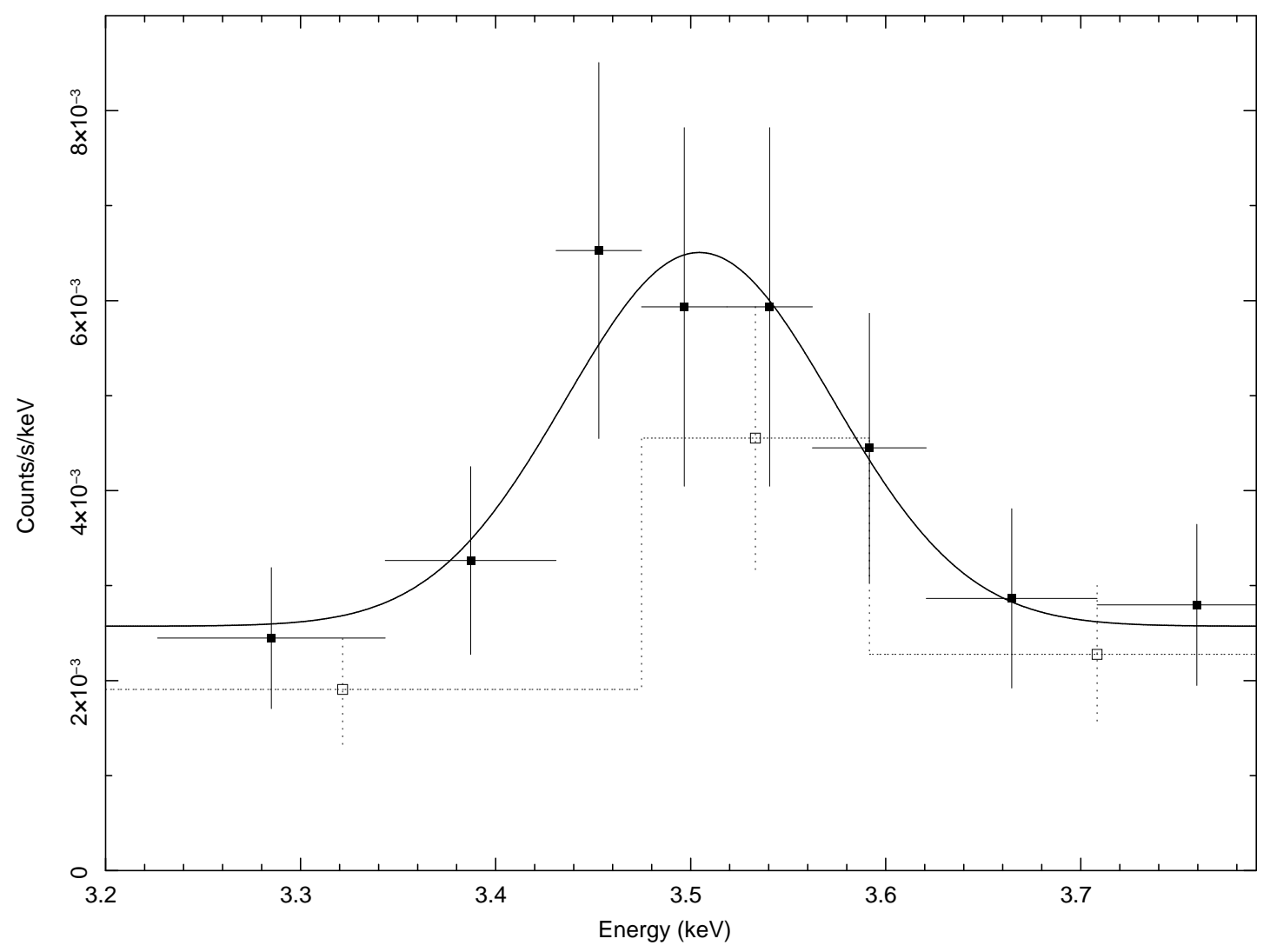

Fig. 3.- Chandra ACIS-S spectra of the pulsar CXO J164710.20-455217 showing the faint feature near $3.5 \mathrm{keV}$. The spectra are rebinned to a minimum of 10 counts per bin. Open squares are from the May 2005 observation $(18.8 \mathrm{ksec})$ and solid squares are from June 2005 (38.5 ksec). The Gaussian fit to the second observation is centered at $\mathrm{E}=3.504 \mathrm{keV}$. The feature contains 23 net counts $[3.4-3.6 \mathrm{keV}]$ above the continuum in the second observation and background is negligible ( $<1$ count in this narrow energy range). 


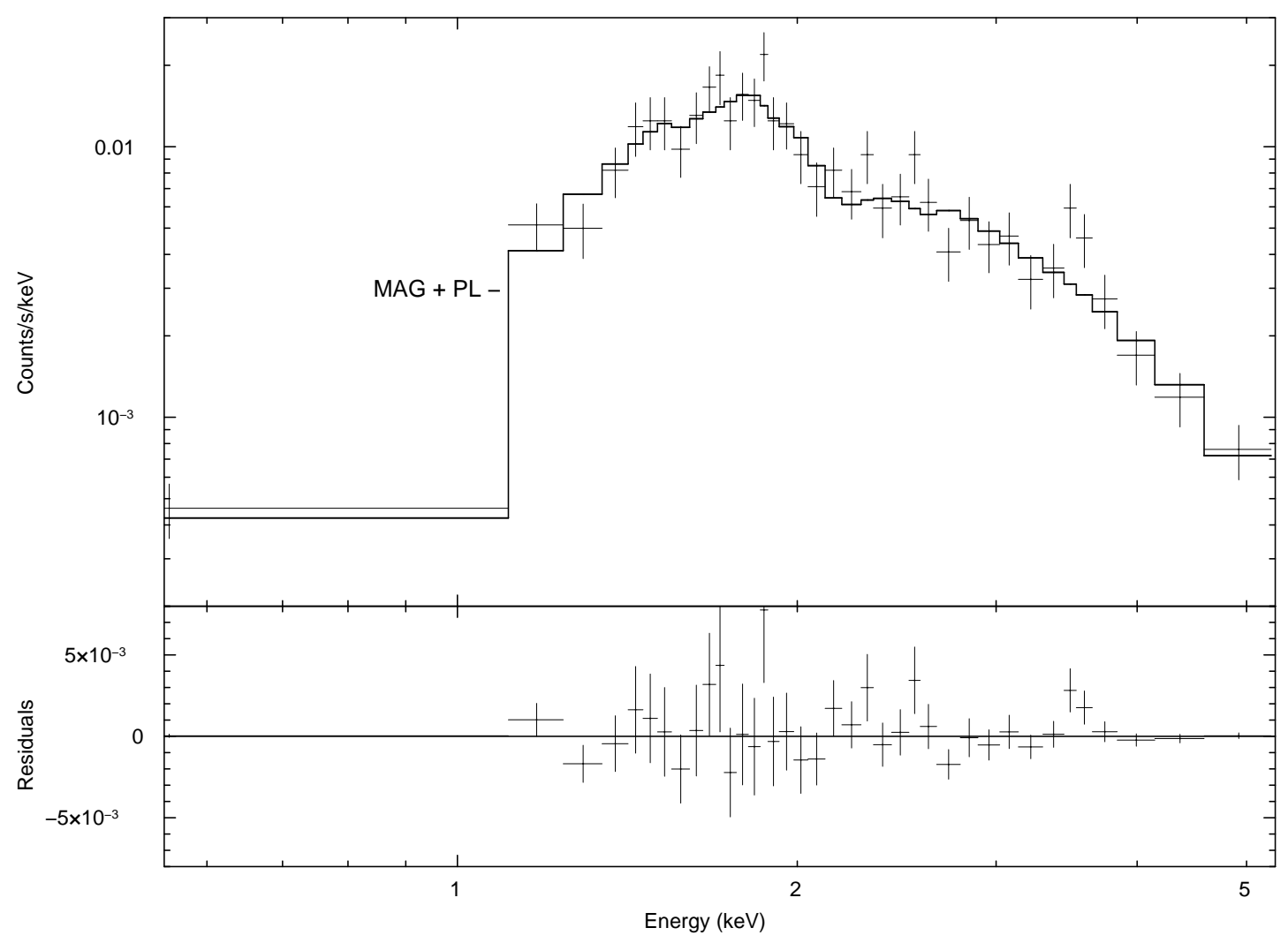

Fig. 4.- Background-subtracted Chandra ACIS-S spectrum of the pulsar CXO J164710.20-455217 obtained on 18-19 June 2005 (834 counts). The spectrum is rebinned to a minimum of 20 counts per bin. The overlaid model is a 2-component magnetar + power-law (model E in Table 1). 


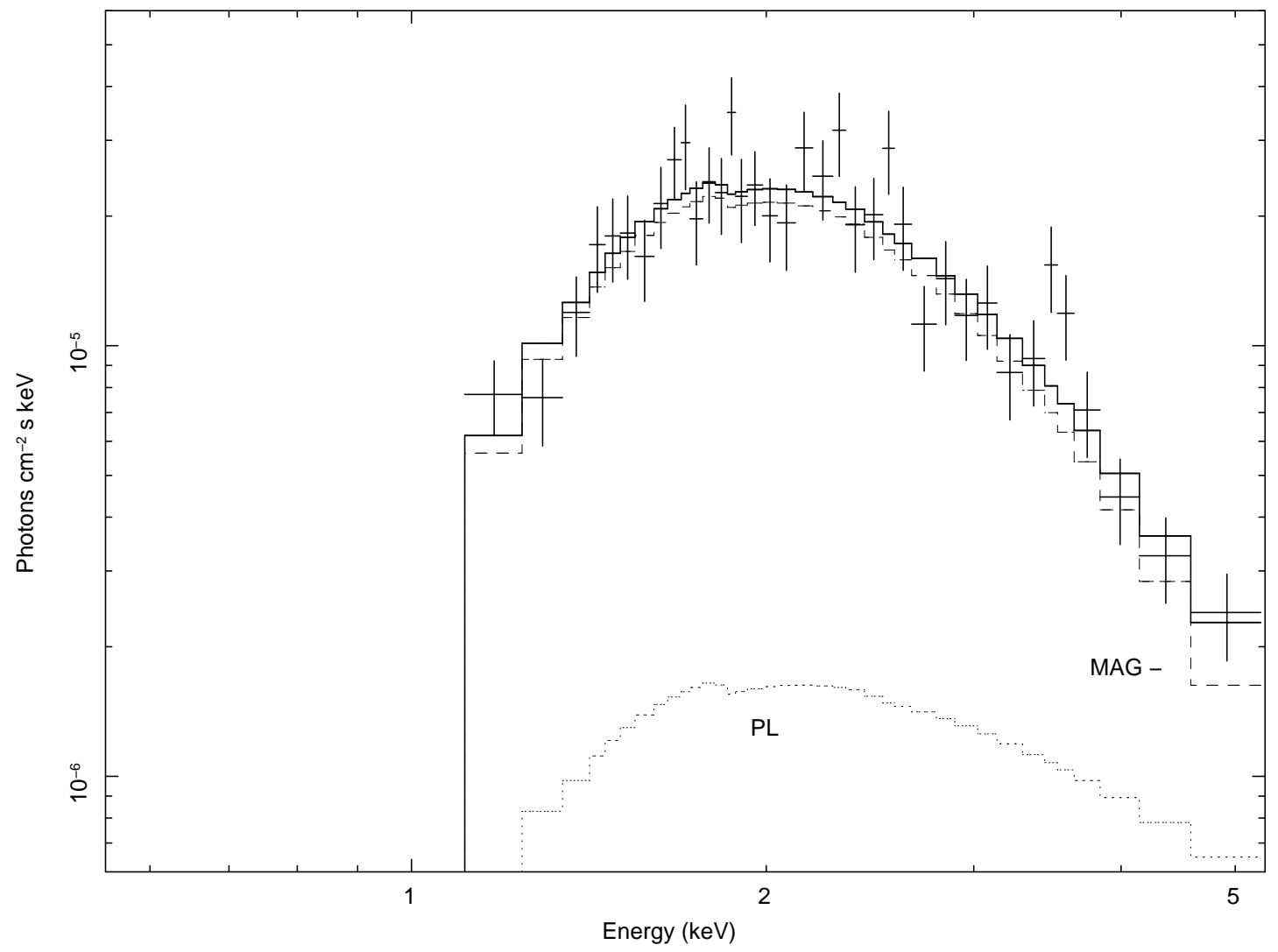

Fig. 5.- Same as Figure 4 showing the unfolded spectrum. 